\title{
A Quality Control Model for Extended Enterprises and Its Implementation
}

\author{
Yongtao Qin, Liping Zhao, Yiyong Yao and Damin Xu \\ State Key Laboratory for Manufacturing Systems Engineering, School of Mechanical \\ Engineering, Xi'an Jiao Tong University, Xi'an 710049, P.R. China \\ qinyt23419@126.com lipingzh@mail.xjtu.edu.cn \\ yyyao@mail.xjtu.edu.cn daminxu@gmail.com
}

\begin{abstract}
Along with the intensification of global competition and the complexity of manufacturing products, cooperation among enterprises becomes more intimately, and the range of quality control extends from internal to the external enterprises. Consequently, previous quality control methods for internal enterprise have been difficult to meet the demands of extended enterprises. To effectively realize the quality control of extended enterprises, it is necessary to research the quality control model which adapt to extended enterprise. To meet the demand of extended enterprises' quality control, in this paper, on the basis of the fractal characteristic of quality control in extended enterprises, based on fractal method, combine with complex networks, quality control fractal network is established by constraint relationship among nodes and node. Base on quality control fractal network, quality control model is constructed by some methods to manipulate and operate node and constraint relationship. Finally, Based agent technology, the implementation method of quality control model is studied to meet the demand of quality control, and it can provide an approach to solve quality control problems for extended enterprises.
\end{abstract}

Keywords: Quality control, Extended enterprise, Manufacturing

\section{INTRODUCTION}

Along with the intensification of global competition and the complexity of manufacturing products, cooperation among enterprises becomes more intimately, the range of quality control extends from internal to external enterprises, these issues engender it more difficult to quality control for extended enterprises in modern networking manufacture, so that the previous methods of intra-enterprise quality control has been difficult to apply to inter-enterprise quality control. In order to control effectively extended enterprise quality, a new model must be established to adapt to the requirement of quality control for extended enterprise [1-7].

Therefore, some researchers in domestic and overseas studied a lot about it, and fractal theory present a new approach to resolve problems in complex systems, but current fractal theory mostly focuses on the study about the quality control of internal enterprises, and with a view to realizing the quality control of extended enterprises, 
meeting the demand of quality control's open and extensibility feature for extended enterprises [8]. In this paper, based on fractal method, firstly quality control model for extended enterprises is established, and the implementation of quality control for extended enterprises is presented in this paper.

\section{QUALITY CONTROL MODEL FOR EXTENDED ENTERPRISE}

\subsection{Fractal Characteristic of Quality Control in Extended Enterprises}

The quality control for extended enterprises integrates the quality control information of internal enterprises which has their own quality control system according to some strategies under the commend of core enterprise, and meet the demand of products quality, exerts overall benefit, so that it has some fractal characteristic in quality control function [9-10].

When the quality control for extended enterprises has been seen as a total unit, the general target of quality control has been decomposed after the extended enterprises chose cooperative partners, and created some executable child quality control units with restricted a certain conditions and associated each other. The child quality control units also could be thought as parent units, and these units' function will be achieved by sub-quality control units' effect. Quality control units can be divided as core enterprises, partner enterprises, manufacturing cell, equipment unit and until the quality control of a specific unit or a part according to the hierarchical structure of extended enterprises. Each unit which can be seen as a relatively independent part to some extent reappears and reduces entirety, and has independent action, adjusting goal, self-similarity feature. Figure 1 show the quality control unit that has common function in extended enterprises.

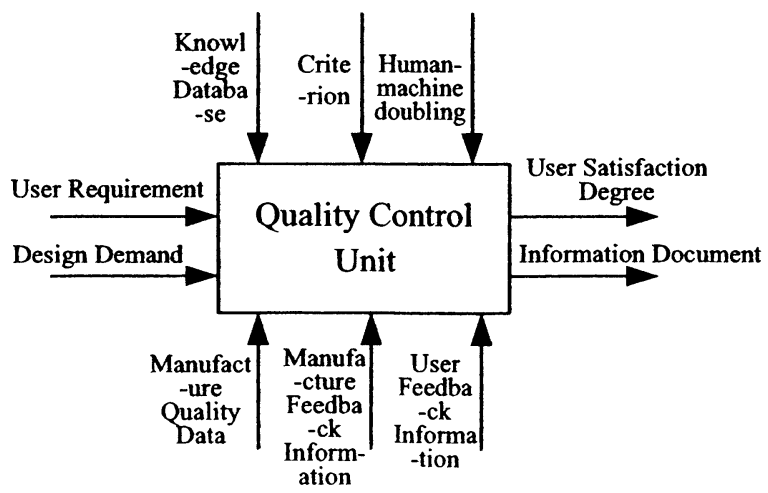

Figure 1. The Quality Control Unit 
Under a certain strategy, quality control system with complex and fantastic characteristic for extended enterprises has been constructed by constraint relationship among nodes on the basis of management or coordination rule among quality control units. An open and extensibility organization structure can be established based this system. The organization structure has self-similarity feature. As a result, quality control in extended enterprises has fractal characteristic.

\subsection{Quality Control Model for Extended Enterprise}

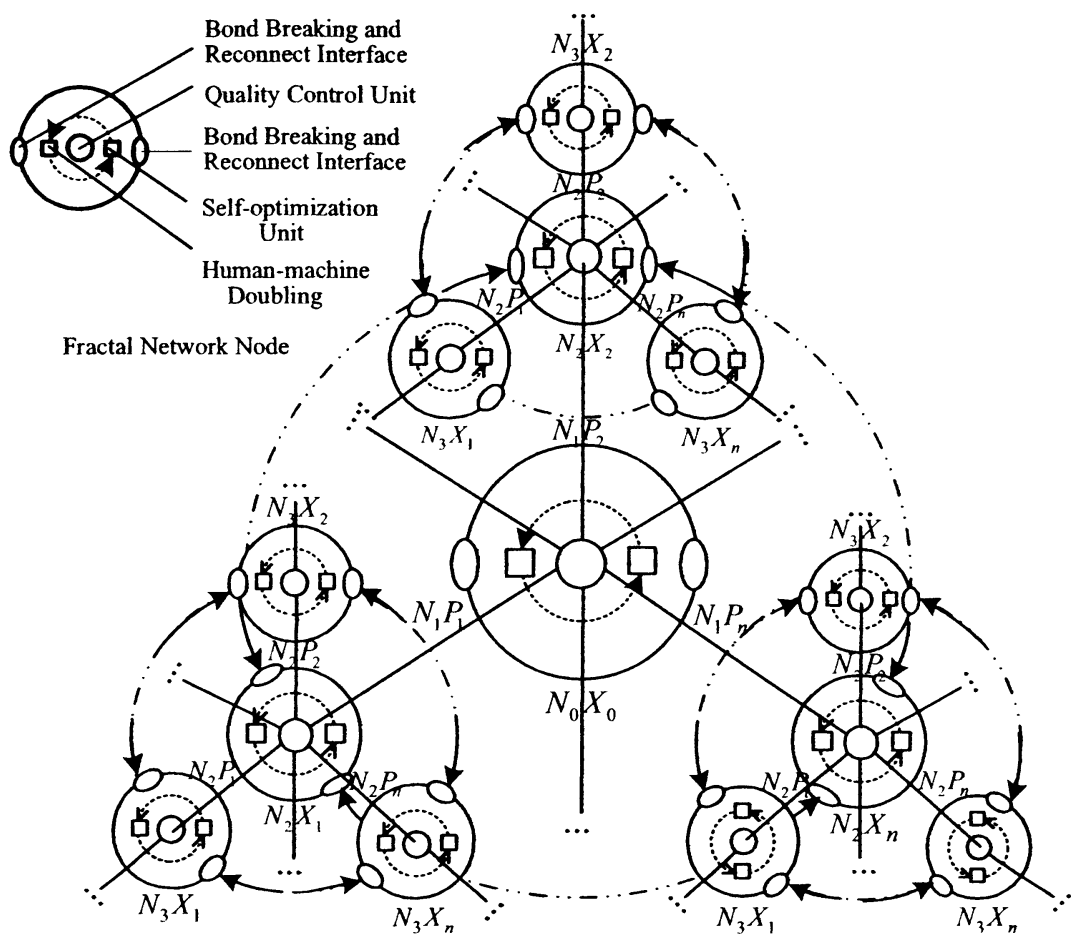

Figure 2. The Quality Control Model for Extended Enterprise

Base on the principle of interest optimization, extended enterprise ally with some cooperative enterprises on the premise of profit maximization, and meet the quality control demand. With view to accurate express quality control state for extended enterprise and research some optimization method, coordinate quality control for better improve quality control effect, further study the rules of quality control for extended enterprise. Therefore, based on fractal method, combined with complex networks, quality control fractal network has been established by constraint relationship among nodes and node. Base on quality control fractal network, quality 
control model is constructed by some method to manipulate and operate node and constraint relationship. Quality control model for extended enterprise is shown in figure 2 .

Real line in figure 2 shows management constraint relationship of upper layer's to lower layer's nodes; dotted line shows constraint relationship of coordinated among same layer's nodes; the constraint relationship can express relationship and connection among nodes, and information's reconstruction. Management constraint of upper layer's to lower layer's nodes and coordinated cooperation constraint among same layer' nodes is unilateral. That is to say the connection among nodes is undirected.

There are definitions for further describe the model:

\section{(1) Node}

The $N_{i} X_{j}$ node is described as below:

$$
N_{i} X_{j}=\left\{N_{i}, X_{j}\right\} \mid 0 \leq i<n, 0<j<m
$$

$N_{i}$ represents the $i$ layer node. $\quad X_{j}$ represents the $j$ node.

The value is decided by actual quality control system, for example, $i=4$ for extended enterprise generally, that is to say extended enterprise is divided into 4 layers, which are core enterprise layer, partner enterprise layer, manufacture cell layer and equipment layer.

Based on quality control function unit, fractal network node is constructed by modularization and object - oriented methodology. The node constitution $(N C)$ is constructed as below:

$$
N C=\left\{N_{i} X_{j}, I Q, O Q, Q C, D S, C T\right\}
$$

$N_{i} X_{j}$ represents the $j$ node in the $i$ layer;

$I Q$ represents input quality information which includes input information by manual work or quality information of other nodes;

$O Q$ represents output quality information which denotes communication information among nodes by unified encapsulation and standardized expression.

$Q C$ represents quality control unit:

$$
Q C=\{G, D, A, S, F\}
$$

G: quality goal analyzing cell; $\quad$ D: quality assignment allocation cell;

S: quality information integration cell; F: quality decision and prediction cell;

A: quality assignment action and monitor cell.

$D S$ represents requirement of quality control, such as standard, user demand, technical condition, production capacity, and so on;

$C T$ represents communication unit which express transmission and acceptance of quality control information among nodes.

(2) Constraint Relationship 
Based on centralized and distributive management strategy, constraint relationship among nodes is constructed:

$$
C B=\left\{R_{i}, C\right\}
$$

$C B$ represents constraint relationship;

$R_{i}$ represents constraint relationship type;

$i$ ranges from 1 to $2, R_{1}$ is the constraint relationship between upper and lower

layer's nodes; $R_{2}$ present the constraint relationship among same layers:

$$
R_{\mathrm{l}}=\left\{R M, N_{i} P_{k}\right\}
$$

$R M$ represents management constraint between upper and lower layer's nodes;

$N_{i} P_{k}$ represents weights of management constraint among layers;

$$
N_{i} P_{k}=\left\{N_{i}, P_{k}\right\} \mid 0 \leq i<n, 0<k<p
$$

$N_{i}$ represents the $i$ layer node. $P_{k}$ represents weight value is $k$.

According to differences in production output, human resource and cooperation condition, management constraint between upper and lower layer's nodes have different weights.

$$
R_{2}=\left\{R L_{l}, D L\right\}
$$

$R L_{j}$ represents coordinated constraint relationship among same layers, value $j$ is 1,2 , or 3 , which represents exclusion relationship, necessity relationship and optional relationship respectively;

$D L$ represents dynamic reconfiguration, value $j$ is 0 or 1 which represents quality control can reconstruct, value $j$ is 2 which represents quality control can not reconstruct.

Based on the construction of node and complex connection created by constraint relationship, fractal network can be established. The network structure has diversity and randomly selectivity, can adjust action by constantly collection feedback information, evaluation spontaneous information received [11-12]. It reflected the self-organization, self-optimization, and self-organization characteristic of network.

Based on fractal network, the quality control model for extended enterprise is constructed by the quality information capsulation based template schemes, or BOM with object-oriented programming, some quality control methods such as SPC, control chart, QFD to manage the quality information among nodes, some optimization algorithms to coordinate the interaction among node such as cooperation, conflict, centralized, and distribution strategy such as constraint logic programming to constraint the quality control relationship among nodes such reconstruction.

\section{IMPLEMENTATION OF QUALITY CONTROL MODEL}




\subsection{Implementation Framework of Quality Control Model}

According to characteristic and requirements of quality control for extended enterprise, based on fractal method, quality control model is established. The model can be implemented through studying implementation method. The implementation framework of quality control for extended enterprise was shown in figure 3.

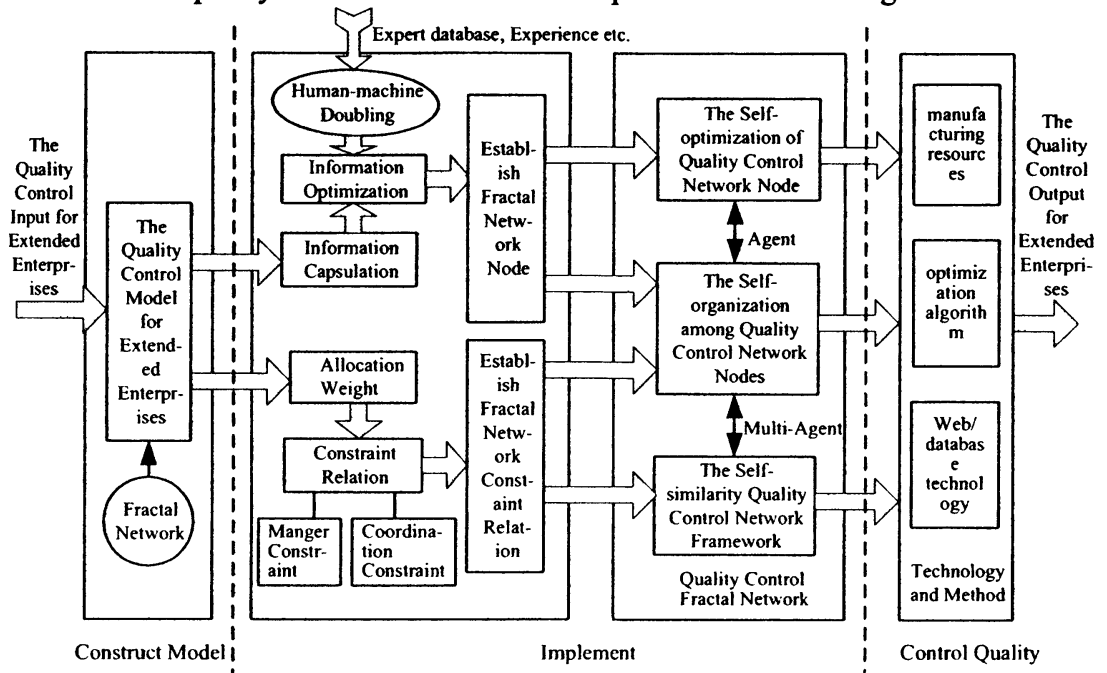

Figure 3. Implementation Framework of Quality Control Model

The framework of quality control model has several steps to realize implementation, as follows:

Firstly, based on fractal network, the quality control model is constructed. Secondly, node is established by information optimization, capsulation, humanmachine doubling, experience, and so on. The template or BOM can be used in information capsulation, and CORBA, XML and ontology can be used in the standard expression of information. Constraint relation among nodes is established by external enterprises structure, product configuration, and so on. Weight allocation, constraint logic programming can be used in constraint's construction. Thirdly, base on agent technology, fractal agent is established to realize the node function, and has selfoptimization characteristic. Multi-agent strategy is established to realize constraint relation by centralized and distribution strategy, discrete particle swarm optimization algorithm, and quality control methods, so that fractal network structure has selfsimilarity, self-organization characteristic. In conclusion, quality control can be achieved by some technologies and methods such as Web and database technology, optimization algorithm, and outer condition, and some manufacturing resources. 


\subsection{The Implementation Method of Quality Control Model}

\subsubsection{Quality Control Fractal Agent}

Because network node is the key and basis of quality control model for extended enterprise, the fractal network node must be established to control quality for extended enterprise firstly. Accordingly, based on quality control unit, quality control fractal agent is constructed by agent technology, modularization and object - oriented methodology so as to realize node function, and has self-optimization characteristic. The structure of quality control fractal agent as illustrated in Figure 4.

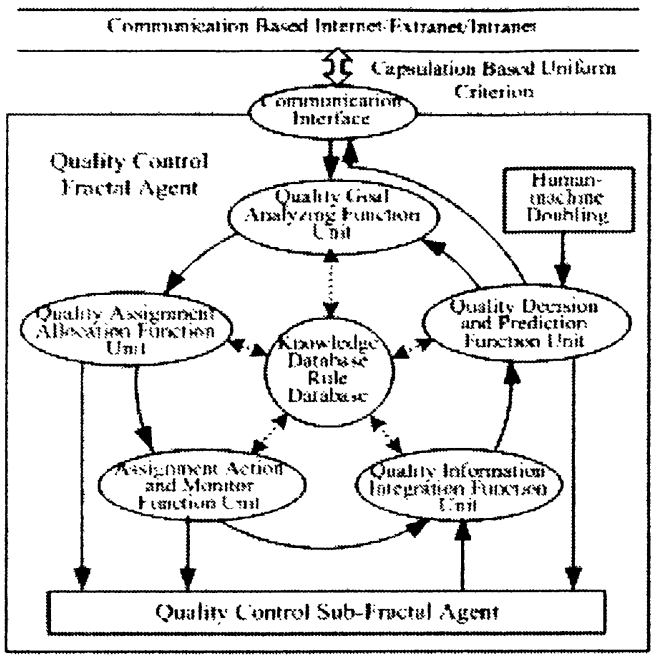

Figure 4. The Structure of Quality Control Fractal Agent

The structure of quality control fractal agent has several processes to realize its functions, as follows:

In the quality control fractal agent, Firstly, quality goal analyzing function unit analyzed the quality information form communication interface, constructed quality control goals and transfer to other units. The input's information is communicated by Internet/Extranet/Intranet, and capsulation based uniform criterion. Secondly the quality assignment allocation function unit allocated and assigned the goals to subagent. Thirdly, assignment action and monitor function unit execute and monitor quality control. Fourthly, quality information integration function unit integrate quality information. Finally, quality decision and prediction function unit can predict and evaluate quality information, and feed back information to quality goal analyzing function unit on the basis of knowledge, rule database and Human-machine doubling, so as to it can modify and accommodate quality control goal and achieve self cycle optimization. Fractal agent is describing:

$$
\text { Fractal agent }=\left\{\sum \text { Unit }_{i}, Q C\right\} \mid i=1,2 \ldots, n
$$

(1) Fractal agent represents quality control fractal agent; 
(2) $Q C$ represents quality control unit;

(3) $\sum$ Unit $_{i}$ represents organization structure, the set consisted of suborganization is a recursion nesting, and may be an organization that possess function such as technology, manufacture. The sub-organizations that constituted fractal agent are labeled sub-fractal agent.

\subsubsection{Logical Structure of Implementation Method}

To the characteristic and demand of quality control for extended enterprise, the quality control method can realize by quality control model constructed for extended enterprise. Firstly, fractal network node is established by agent technology, CORBA, $\mathrm{XML}$, ontology. Secondly, constraint relationship is produced on the basis of Soft Bus among quality control fractal agents. Eventually quality control fractal network is built to control quality. The logical structure of implementation method is presented in figure 5.

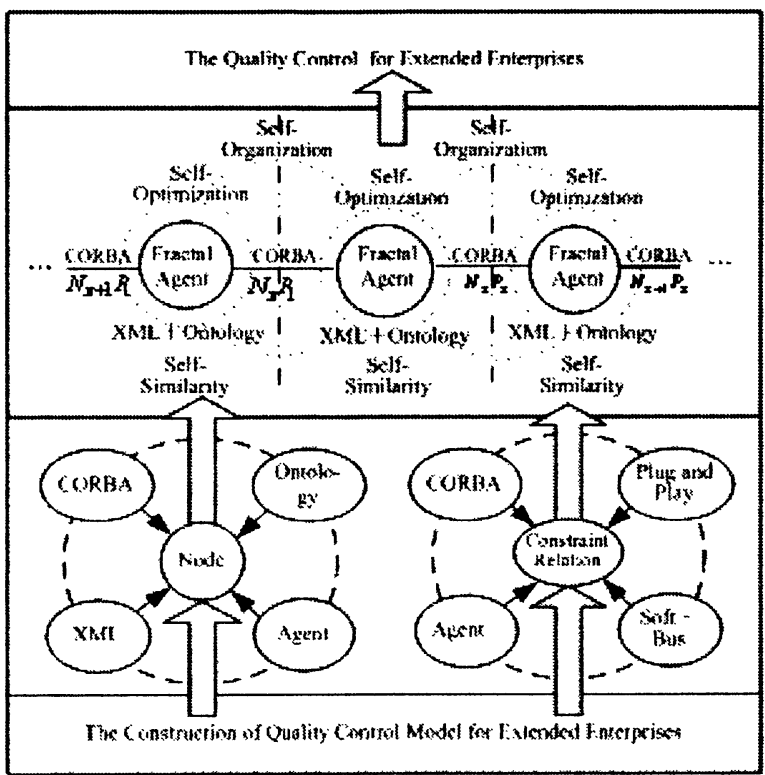

Figure 5. The Logical Structure of Implementation Method

Constraint relationship between management and coordinated are established by making use of "soft-bus" structure that is produced by CORBA and a certain control strategy on the basis of the characteristic of enterprises. Fractal agents are connected by Plug and Play with the registration function of network. Then, based on database and information environment by Intranet/ Extranet/Internet, ontological expression by XML, communication among agents, CORBA's support to transmission protocol, so 
that quality control information that transmit among agents can smoothly communicate through the environment of heterogeneous systems and protocol[ 13-15].

Constraint relationship among fractal agents is established by "soft bus" structure and "Plug and Play" mode in self-organization network. Fractal network structure of quality control which has self-similarity, self-optimization, and selforganization is constructed by network complicated evolution. On the basis of open and extensibility feature of the fractal network, using Web technology by Internet/Extranet/Intranet to assure Real-time control, cooperation based the fractal network, the quality of extended enterprises can be control which can implement quality establishment, allocation, and action from core enterprise layer to equipment layer, and achieve quality evaluation, judgment, and prediction from equipment layer to core enterprise layer.

\section{CONCLUSIONS}

In this paper, on the base of the self-similarity Characteristicistics in the quality control of extended enterprises, based on fractal method and complex networks, depended on constraint relationship among nodes and node, the self-similarity, selfoptimization, self-organization fractal network structure is established. Based on fractal network, the quality control model for extended enterprise is constructed by quality information capsulation based template schemes or BOM with object-oriented programming, some quality control methods to manage the quality information among nodes and manipulate constraint relationship among nodes, some optimization algorithms to coordinate the interaction among node, centralized and distribution strategy to constraint the quality control relationship among nodes. Finally based on agent technology, the implementation method of quality control model is studied by CORBA, $\mathrm{XML}$, ontology, and so an, so that this model has open and extensibility feature for extended enterprises, can meet the demand of quality control, and it brings an approach to solve quality control problems for extended enterprises.

\section{ACKNOWLEGEMENTS}

This work was supported by grant No. 2006AA04Z149 form the National High Tech. R\&D Program for Contemporary Manufacturing Integrated Technology, China.

\section{REFERENCES}

1. J.L. Paris and H. Pierreval, Modelling and Simulation of Manufacturing Systems and Extended Enterprises, Simulation Modeling Practice and Theory. Volume 15, pp.111$112,(2007)$. 
2. F. Chan, R. Swarnkar, and M. Tiwari, Infrastructure for co-ordination of multi-agents in a network-based manufacturing system, The International Journal of Advanced Manufacturing Technology. Volume 31, pp.1028-1033, (2007).

3. F. Linington, A Unified Behavioural Model and a Contract Language for Extended Enterprise, Data \& Knowledge Engineering. Volume 51, Number 1, pp.5-29, (2004).

4. Y. Hu, T. Yu, L. Liu, and H. Sun, Knowledge Enterprise: Intelligent Strategies in Product Design, Manufacturing, and Management, in Proc. of International Federation for Information Processing (IFIP) eds. K. Wang, G. Kovacs, M. Wozny, and M. Fang (Springer: Boston, MA, 2006), pp.902-907.

5. M. Palaciosa, E. Alvarez, M. Alvarez, and J. Santamaria, Lessons learned for building agile and flexible scheduling tool for turbulent environments in the extended enterprise, Robotics and Computer-Integrated Manufacturing. Volume 22, pp.485-492, (2006).

6. O. Bayazita and B. Karpak, An analytical network process-based framework for successful total quality management (TQM): An assessment of Turkish manufacturing industry readiness, International Journal Production Economics. Volume 105, pp.79-96, (2007).

7. W. Wei, L. Zhao, and Y. Yao, Collaborative quality tracking model based on workflow, Computer Integrated Manufacturing Systems. Volume 12, Number 10, pp.1586-1590, (2006).

8. S. Zhang and B. Wang, Managing Access in Extended Enterprise Networks Web ServiceBased, Lecture Notes in Computer Science. Volume 3251, pp.963-966, (2004).

9. H. Dong, 'D. Liu, Y. Zhao, and Y. Chen, A novel Approach of Networked Manufacturing Collaboration: Fractal Web-based Extended Enterprise, The International Journal of Advanced Manufacturing Technology. Volume 26, Number 11, pp.1436-1442, (2005).

10. M. Shin, Y. Cha, K. Ryu, and M. Jung, Conflict Detection and Resolution for Goal Formation in the Fractal Manufacturing System, International Journal of Production Researc. Volume 44, Number 3, pp.447-465, (2006).

11. P. Melin and O. Castillo, An intelligent hybrid approach for industrial quality control combining neural networks, fuzzy logic and fractal theory, Information Sciences. Volume 177, pp.1543-1557, (2007).

12. Y. Yao, G. Lin, and A. Trappey, Using Knowledge-Based Intelligent Reasoning to Support Dynamic Equipment Diagnosis and Maintenance, International Journal of Enterprise Information Systems. Volume 2, Number 1, pp.17-31, (2006).

13. M. Lim and D. Zhang, An integrated agent-based approach for responsive control of manufacturing resources, Computers \& Industrial Engineering. Volume 46, pp.221-232, (2004).

14. O. Lopez-ortega and M. Ramirezr, A STEP-based manufacturing information system to share flexible manufacturing resources data, Journal of Intelligent Manufacturing. Volume 16, pp.287-301, (2005).

15. M. Sakthivel, S. Devadasan, S.R. Raman, and S. Sriram, Design and Development of a Quality Management Information System, International Journal of Enterprise Information Systems. Volume 2, Number 4, pp.18-37, (2006). 